\title{
Transient sub-surface uplift of a floating ice sheet
}

\author{
J. P. Dempsey and Z. G. Zhao \\ Department of Civil and Environmental Engineering, Clarkson University, \\ Potsdam, NY 13699-5710, U.S.A.
}

\begin{abstract}
In this paper, the transient response of an ice sheet subjected to sudden uplift is investigated. The ice sheet is modeled as an infinite thin plate undergoing small deflections that is being pushed vertically upwards by a rigid flatended cylinder. The water underneath the ice sheet is modeled using incompressible potential flow theory. For a given motion of the cylinder, the current problem is reduced to a Volterra integral equation of the first kind for the indentation force. It is solved by transforming the governing equation into an integro-differential equation. For the physical quantities of interest, comparisons are made with related experiments.
\end{abstract}

\section{INTRODUCTION}

Many of the civilian and military operations in the Arctic and Antarctic regions, as well as on inland lakes and rivers, subject floating ice covers to dynamic loading conditions, corresponding to the impact loads of landing airplanes, submarine surfacings, moving surface loads and pulsating loads of heavy machinery. The problem in the title is germane to the submarine surfacing problem and is motivated by recent experiments at the U.S. Army Cold Regions Research and Engineering Laboratory (CRREL) in Hanover, NH (Sodhi, 1989; McGilvary and others, 1990). The objective of this study is to investigate the transient response for a given dynamic uplift. The ice sheet is modeled as a thin plate undergoing small deflections, which is pushed vertically upwards by a flatended cylinder of radius $c^{\prime}$. It is assumed that the ice sheet is at rest prior to contact. The cylinder is assumed to contact the bottom surface of the ice sheet on the circular edge of the flat end only (Fig. 1), and the water is modeled using incompressible potential flow theory.

In the case of forced vibrations or dynamic loading, an additional bottom-surface pressure is imparted by the water as the water mass is accelerated. Holl (1950) considered the forced vibration of thin plates resting on elastic foundations of the Winkler type, but did not include the effects of the acceleration of the water. Kheysin (1967) and Nevel (1970) considered the vibration of a floating ice sheet, including the acceleration of the water. The latter analysis treated a time-varying load uniformly distributed over a circular area of unchanging radius. Sodhi (1989) recently conducted a series of small-scale tests in which floating ice sheets were pushed vertically upwards by vertical cyclinders having different end shapes (flat, truncated-conical and conical) and different diameters $(76,152$ and $305 \mathrm{~mm})$. In this

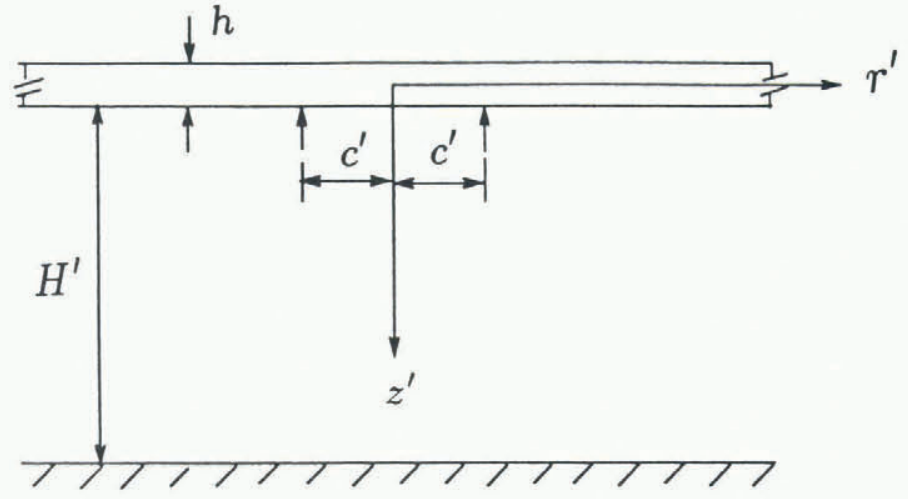

Fig. 1. Problem coordinates and loading configuration. The uplift force exerted by the flat end of the cylinder is approximated by a timedependent ring load.

series of tests, the ice sheets were subjected to vertical uplift and indentation at essentially constant velocity. The experiments reported by Sodhi (1989) are interesting in that the form of the dynamic loading is amenable to an analytical treatment, if only for short time periods. The loading by the flat-ended cylinders can be idealized as a ring load, and the simplifying advantages of axisymmetry can be utilized. In this paper, the expressions for the imposed motion of the ice sheet - see Equation (3) - are the same as those used by McGilvary and others (1990).

Note that the simple model of a Winkler foundation has often been used to analyze the strength and deflection of floating ice sheets. In this type of foundation, the pressure on the underside of the ice sheet is assumed to be directly proportional to the downward deflection of the ice sheet (the first term in Equation (7)). However, 
the resisting force due to buoyancy is, in fact, rigorously represented by the Winkler model only for the case of static or quasi-static loading conditions.

Urea model ice was used in the basin by Sodhi (1989). This particular model ice is used to represent sea ice since it has a very similar microstructure but, by design, for modeling purposes it is very weak. Urea model ice is, with fair surety, substantially more plastic and deformable than real sea ice. The use in this paper, therefore, of an undamped non-viscoelastic model is more of an idealization than if the tests were done with freshwater ice, for instance. However, the model presented should still be a close approximation to reality up to times for which the small deflection thin-plate theory solution is valid.

A major difficulty confronted by analysts in attempting to compare theoretical predictions with experimental results is the importance of the characteristic length $l$ - see Equation (8c) - and the fact that it is measured under quasi-static loading conditions whilst the experiments are rapid. A further complication is the fact that an infinite ice sheet subjected to axisymmetric deformations is discussed in this paper, whereas the basin in which Sodhi's experiments were conducted is, in fact, not only finite but also rectangular (the finite in-plane dimensions would also possibly influence the characteristic length measurements). However, since the lowest ratio of the half-basin width to the measured characteristic length is 3.66, note the comment by Sodhi (1989) that "This ratio, being more than 3 , means that the effect of the basin walls is negligible on the results of our experiments."

\section{FORMULATION}

In the problem under consideration, the ice sheet is assumed to be at rest prior to contact, and there is no applied force prior to contact. Furthermore, the uplift force must be positive or zero (no tension at the contact line is allowed). The motion of the ice sheet is therefore governed by

$$
\rho_{\mathrm{i}} h \frac{\partial^{2} w^{\prime}}{\partial t^{2}}+D \nabla^{4} w^{\prime}+p_{0}^{\prime}=\frac{P^{\prime}(t)}{2 \pi c^{\prime}} \delta\left(r^{\prime}-c^{\prime}\right),
$$

where $\rho_{i}$ is the mass density of the ice sheet, $h$ is its thickness, $w^{\prime}$ its vertical displacement and $\nabla^{2}=\partial^{2} / \partial r^{\prime 2}+$ $\left(1 / r^{\prime}\right) \partial / \partial r^{\prime}$; furthermore $\nabla^{4}=\nabla^{2} \nabla^{2}, D$ is the flexural rigidity, $P^{\prime}(t)$ the unkown time-varying force uniformly distributed on the circle $r^{\prime}=c^{\prime}$ (a ring load), $\delta()$ the Dirac delta function and $p_{0}^{\prime}$ the pressure exerted by the water reaction. The initial and boundary conditions are

$$
\begin{gathered}
w^{\prime}=0 \text { and } \dot{w}^{\prime}=0, \text { for } t \leq 0, \quad 0 \leq r^{\prime}<\infty ; \\
P^{\prime}(t)=0, \text { for } t<0 ; P^{\prime}(t) \geq 0 \text { for } t \geq 0
\end{gathered}
$$

In the experiments by Sodhi (1989), the cylinders were moving upwards at a constant velocity before contact. Conservation of momentum requires that the cylinders experience some decelaration, albeit slight. The forced displacement of the plate, upwards in this study, is therefore expressed in terms of the imposed velocity using the expressions adopted by McGilvary and others (1990)

$$
\begin{aligned}
& w^{\prime}\left(c^{\prime}, t\right)=\left\{\begin{array}{lr}
-h_{1}\left[\left(t / t_{0}\right)^{3}-0.5\left(t / t_{0}\right)^{4}\right]\left(t_{0} / T\right), & 0 \leq t \leq t_{0}, \\
-h\left[\left(t / t_{0}\right)-0.5\right]\left(t_{0} / T\right), & T \geq t \geq t_{0},
\end{array},\right. \\
& \dot{w}^{\prime}\left(c^{\prime}, t\right)= \begin{cases}-v_{\infty}\left[3\left(t / t_{0}\right)^{2}-2\left(t / t_{0}\right)^{3}\right], & 0 \leq t \leq t_{0}, \\
-1, & T \geq t \geq t_{0},\end{cases}
\end{aligned}
$$

in which

$$
\begin{aligned}
T & =h / v_{\infty}, & t_{0} & =0.05 \mathrm{~s}, \\
h & =0.076 \mathrm{~m}, & v_{\infty} & =0.038 \mathrm{~ms}^{-1}
\end{aligned}
$$

and $v_{\infty}$ is the velocity of the cylinder prior to contact. The time constant $T=h / v_{\infty}$ is the time taken by the cylinder to traverse the plate thickness if no deceleration on contact takes place. Since the cylindrical indentors in the physical experiments attempt to move upwards at a constant velocity $v_{\infty}, T$ is a useful parameter for the solution obtained in this paper. With the limitations of thin-plate theory being operative (small deformations), the solutions presented are therefore necessarily restricted to time $t<T$.

The bottom-surface pressure due to the acceleration of the water is treated by assuming that the motion of the water is governed by potential flow theory (Kheysin, 1967; Nevel, 1970). To this end, let $\phi^{\prime}$ be the corresponding water potential function. Assuming that the water is incompressible, conservation of water mass requires that

$$
\widehat{\nabla}^{2} \phi^{\prime}=0,
$$

where $\widehat{\nabla}^{2}=\nabla^{2}+\partial^{2} / \partial z^{\prime 2}$. The water velocity vector $\mathbf{v}$ is the gradient of the potential function $\phi^{\prime}$. At the deformed ice-water interface $z^{\prime}=w^{\prime}+h / 2$, and the vertical velocity of the water is equal to the vertical velocity of the ice. Assuming that the depth $H^{\prime}$ of the water under the ice sheet is a constant, the vertical velocity at $z^{\prime}=\left(H^{\prime}+h / 2\right)$ is zero. As $r^{\prime}$ approaches infinity, the velocity is also zero. Since $\phi^{\prime}$ is only determined up to a constant, $\phi^{\prime}$ is set equal to zero as $r$ approaches infinity. $\phi^{\prime}$ must therefore satisfy the conditions

$$
\begin{gathered}
\left.\frac{\partial \phi^{\prime}}{\partial z^{\prime}}\right|_{z^{\prime}=w^{\prime}+h / 2}=\frac{\partial w^{\prime}}{\partial t},\left.\quad \frac{\partial \phi^{\prime}}{\partial z^{\prime}}\right|_{z^{\prime}=H^{\prime}+h / 2}=0, \\
\left.\phi^{\prime}\right|_{r^{\prime} \rightarrow \infty}=0 .
\end{gathered}
$$

The expression for $\phi^{\prime}$ is determined in the next section from Equations (5) and (6) using Hankel transforms. The pressure $p_{0}^{\prime}$ in Equation (1) may then be determined from Bernoulli's equation, assuming that the water has no viscosity and that, as $r^{\prime}$ approaches infinity, $\mathbf{v} \cdot \mathbf{v}=0, p_{0}^{\prime}=\rho_{\mathrm{w}} g w^{\prime}$, and $\phi^{\prime}=0$,

$$
p_{0}^{\prime}=\rho_{\mathrm{w}} g w^{\prime}-\frac{1}{2} \rho_{\mathrm{w}} v^{2}-\left.\rho_{\mathrm{w}} \frac{\partial \phi^{\prime}}{\partial t}\right|_{z^{\prime}=w^{\prime}+h / 2} .
$$

The second term on the righthand side is usually omitted because of its nonlinearity and because, for problems of this type, the resulting approximation is accurate. 


\section{SOLUTION}

Equations (1) and (5), and their initial and boundary conditions (Equations (2), (3), and (6)) are best cast in terms of non-dimensional quantities and coordinates. Therefore, let

$$
\begin{aligned}
& (z, w)=\left(z^{\prime}, w^{\prime}\right) / h, \quad(H, r, c)=\left(H^{\prime}, r^{\prime}, c^{\prime}\right) / l \\
& p_{0}=p_{0}^{\prime} / \rho_{\mathrm{w}} g h, \quad \mu=\rho_{\mathrm{i}} h / \rho_{\mathrm{w}} l, \quad \tau=t \sqrt{g / l} \\
& P(\tau)=P^{\prime}(\tau) 2 \pi \rho_{\mathrm{w}} g l^{2} h, \quad \text { and } \quad l=\left(D / \rho_{\mathrm{w}} g\right)^{1 / 4} .
\end{aligned}
$$

In Equation (8c), $l$ denotes the characteristic length. The use of this length parameter dates back to Hertz (1884) and especially Westergaard (1926); the importance of its use in ice engineering is illustrated by the fact that it is one of the few variables consistently and independently measured in model basin tests (Sodhi and others, 1982). In model basin testing, the characteristic length is measured instead of the modulus of elasticity or the flexural rigidity. In Equation (8), the length normalizations are carried out in terms of either the ice thickness $h$ or characteristic length $l$.

The solution procedure requires the introduction of a zeroth order Hankel transform with respect to $r$, viz.,

$$
\begin{gathered}
H\{f\}=\bar{f}(\gamma, \tau)=\int_{0}^{\infty} r f(r, \tau) J_{0}(\gamma r) \mathrm{d} r, \\
H^{-1}\{\bar{f}\}=f(r, \tau)=\int_{0}^{\infty} \gamma \bar{f}(r, \tau) J_{0}(\gamma r) \mathrm{d} \gamma .
\end{gathered}
$$

Using Hankel transforms Equations ( $9 \mathrm{a}$ and b), Equations (5) and (6) are solved for the case that $H^{\prime}>h$. The Hankel transform of the linearized Equation (7) now gives

$$
\overline{p_{0}}=\bar{w}+\frac{\partial^{2} \bar{w}}{\partial \tau^{2}} \frac{1}{\gamma \tanh \gamma H} .
$$

Applying the same Hankel transform to Equation (1), and inserting Equation (10), the governing equation is reduced to

$$
\left(\mu+\frac{1}{\gamma \tanh \gamma H}\right) \frac{\partial^{2} \bar{w}}{\partial \tau^{2}}+\left(1+\gamma^{4}\right) \bar{w}=J_{0}(\gamma c) P(\tau) .
$$

The solution of Equation (11) for $\bar{w}$ is a Duhamel integral. Using the inverse Hankel transform, the general expression for the displacement follows as

$$
\begin{aligned}
w(r, \tau)= & \int_{0}^{\infty} \beta \gamma \frac{J_{0}(\gamma c) J_{0}(\gamma r)}{1+\gamma^{4}} \mathrm{~d} \gamma \\
& \int_{0}^{\tau} P(u) \sin [\beta(\tau-u)] \mathrm{d} u,
\end{aligned}
$$

where

$$
\beta^{2}=\left(1+\gamma^{4}\right) / \mu_{\gamma}, \quad \mu_{\gamma}=\mu+1 / \gamma \tanh (\gamma H) .
$$

If the ice sheet is pushed up by a cylinder with the velocity as defined in Equation (3b), the dynamic indentation problem is reduced to a Volterra integral equation of the first kind. Numerical solutions of such equations are difficult to obtain; a solution $P(\tau)$ may not exist for every form of uplift velocity that could be specified, and when one attempts to construct one, it may not be unique, or it may be difficult to determine it accurately (Baker, 1977). Fortunately, the form of $w(c, \tau)$ specified by McGilvary and others (1990) is such that $w(c, 0)=0$ and $\dot{w}(c, 0)=0$. The original integral equation is thus transformable into an integro-differential equation, viz.,

$$
w(c, \tau)=P(\tau) \bar{K}(\tau, \tau)-\int_{0}^{\tau} \frac{\partial P(u)}{\partial u} \bar{K}(\tau, u) \mathrm{d} u,
$$

where

$$
\bar{K}(\tau, u)=\int_{0}^{\infty} \frac{\gamma J_{0}^{2}(\gamma c)}{1+\gamma^{4}} \cos [\beta(\tau-u)] \mathrm{d} \gamma .
$$

Employing a direct quadrature method, Equation (14) can be solved for $P(\tau)$. Once the load $P(\tau)$ is evaluated, the temporal and spatial distributions of the displacement can be calculated from Equation (12). The moment can be found from

$$
\begin{aligned}
& \frac{M_{r}^{\prime} l^{2}}{h D}=-\left(w_{, r r}+\frac{\nu}{r} w_{, r}\right), \\
& \frac{M_{0}^{\prime} l^{2}}{h D}=-\left(\nu w_{, r r}+\frac{1}{r} w_{, r}\right),
\end{aligned}
$$

where ()$, r$ represents the derivative with respect to $r$, Once the temporal and spatial distribution of displacement is obtained, $M_{r}^{\prime}\left(r^{\prime}, t\right)$ and $M_{0}^{\prime}\left(r^{\prime}, t\right)$ can be evaluated numerically. The maximum bending stresses follow as

$$
\sigma_{r}=\frac{6 M_{r}^{\prime}}{h^{2}}, \quad \sigma_{0}=\frac{6 M_{\theta}^{\prime}}{h^{2}} .
$$

Similarly, the shear force can be evaluated from

$$
\frac{Q^{\prime} l^{3}}{h D}=-\frac{\mathrm{d}}{\mathrm{d} r}\left(w_{, r r}+\frac{1}{r} w_{, r}\right) .
$$

To avoid convergence problems, Equation (18) is expressed in the alternative form

$$
\frac{Q^{\prime} l^{3}}{h D}=\frac{l^{2}}{h D(1+\nu)} \frac{\mathrm{d}}{\mathrm{d} r}\left(M_{r}^{\prime}+M_{0}^{\prime}\right)
$$

which can be evaluated numerically using finite differences.

\section{RESULTS AND DISCUSSION}

The velocity of the indentor is fixed at $v_{\infty}=0.038 \mathrm{~m} \mathrm{~s}^{-1}$ as in Sodhi's experiments (1989). The ratio of water depth to the thickness of the ice sheet is set to be $H^{\prime} / h=40$. At this velocity, if the thickness of the ice sheet is $0.076 \mathrm{~m}$, the deflection $w^{\prime}\left(c^{\prime}, t\right)$ approaches the thickness $h$ at approximately $t=2 \mathrm{~s}$. Within the limitations of small deflection thin-plate theory, $w^{\prime}\left(c^{\prime}, t\right) \leq h / 2$ is recommended. The characteristic lengths (Equation (8c)) are reduced to $85 \%$ of the values found in Sodhi's experiments (McGilvary and others, 1990).

Figure 2 shows the uplift load versus time, corresponding to test numbers 62,63 and 93 in Sodhi's experi- 


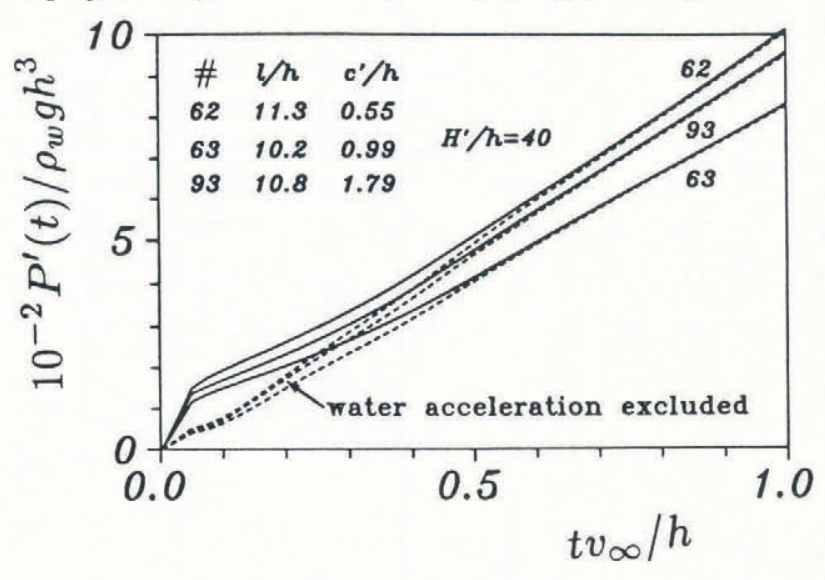

Fig. 2. Uplift load versus time corresponding to the test numbers 62, 63 and 93 in Sodhi's experiments. The dotted lines are applicable if the hydrodynamic reaction is excluded. The foundation is then approximated as a Winkler medium.

ments. The non-dimensional time and the uplift load are expressed as $t v_{\infty} / h$ and $10^{-2} P^{\prime}(t) / \rho_{w} g h^{3}$, respectively. In these three cases the displacement of the indentor reaches $0.43,0.46$ and $0.43 h$, corresponding to the test numbers 62,63 and 93 , respectively. The theoretically determined uplift loads then equal the respective failure loads found by Sodhi (1989) in the associated experiments. However, the associated deflections recorded by Sodhi (1989) were much higher than either $0.43 \mathrm{~h}$ or $0.46 h$.

If the inertia of the ice sheet is deleted from the governing equation, the results are very nearly the same as when the mass of the ice sheet is included, and the corresponding curves are in fact identical for the above three cases. In other words, the inertial force of the ice sheet is negligible compared to the inertial force of the water underneath the ice sheet. Therefore, the ice sheet can effectively be treated as a massless plate with flexural rigidity, $D$. On the other hand, if the dynamic water reaction is excluded, i.e. the water underneath the ice sheet is treated as a Winkler foundation, the corresponding results are shown in Figure 2 with dotted lines. For short times $\left(t v_{\infty} / h \leq 0.5\right)$, the dotted lines are separated from the short ones, for later times $\left(t v_{\infty} / h \rightarrow 1.0\right)$, when

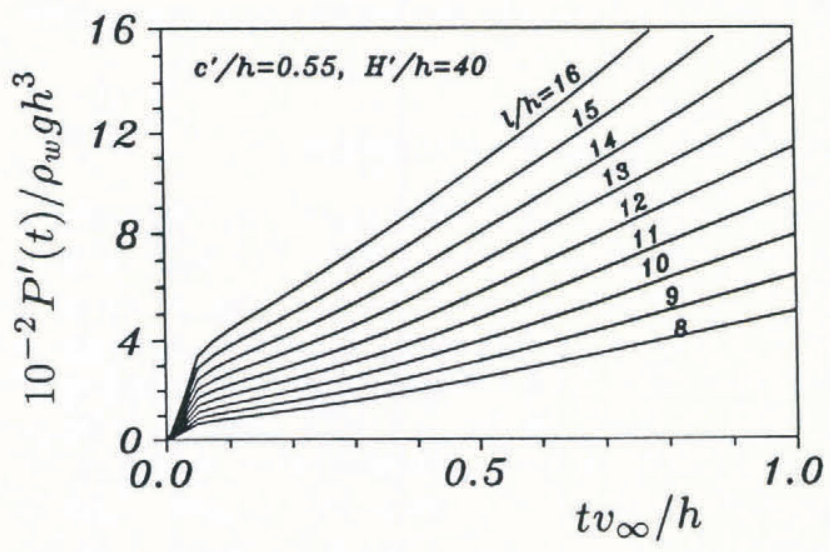

Fig. 3. Influence of the characteristic length on the uplift load.

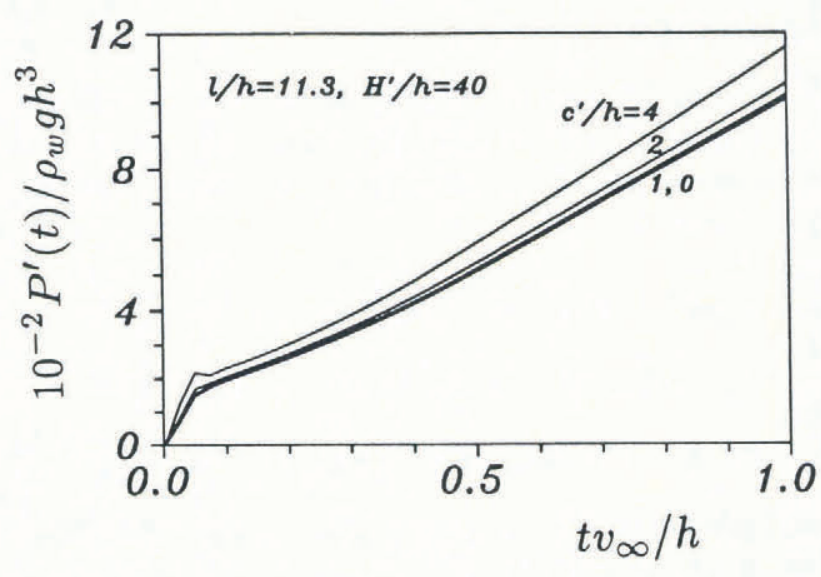

Fig. 4. Influence of the indentor radius on the uplift load.

failure has already occurred, these two sets of curves coincide. The results including the dynamic water reaction forecast a significantly higher uplift load than those excluding it, particularly at early times.

Given the degree of uncertainty regarding the true value for the characteristic length under the dynamic conditions in Sodhi's tests, Figure 3 shows the influence of the characteristic length on the uplift load. The load is steadily increasing as $l / h$ increases. For a given thickness $h$, a larger value of $l / h$ corresponds to a higher value of Young's modulus, which therefore correlates with a larger uplift load.

The influence of the cylinder radius on the uplift load is shown in Figure 4. For $c^{\prime} / h \leq 1.0$, the difference in the uplift load for various values of $c^{\prime} / h$ is negligible and the results are very close to the solution under a concentrated load; whereas for $c^{\prime} / h>1.0$, the difference becomes significant. In Sodhi's experiments, three indentor radii, $0.038,0.076$ and $0.152 \mathrm{~m}$ were used. The influence of the relative water depth on the uplift load has been mentioned previously; only if the ratio $H^{\prime} / h$ is large enough will the above formulation be accurate. In Sodhi's experiments, the range of $H^{\prime} / h$ is $30-45$. The numerical results presented here are accurate for $H^{\prime} / h>5$.

Figure 5 shows the maximum bending stresses at $r=0$ versus time for three test numbers 62,63 and 93. The maximum stress occurs at the center of the flatended cylinder. At $r=0$, the maximum bending stresses

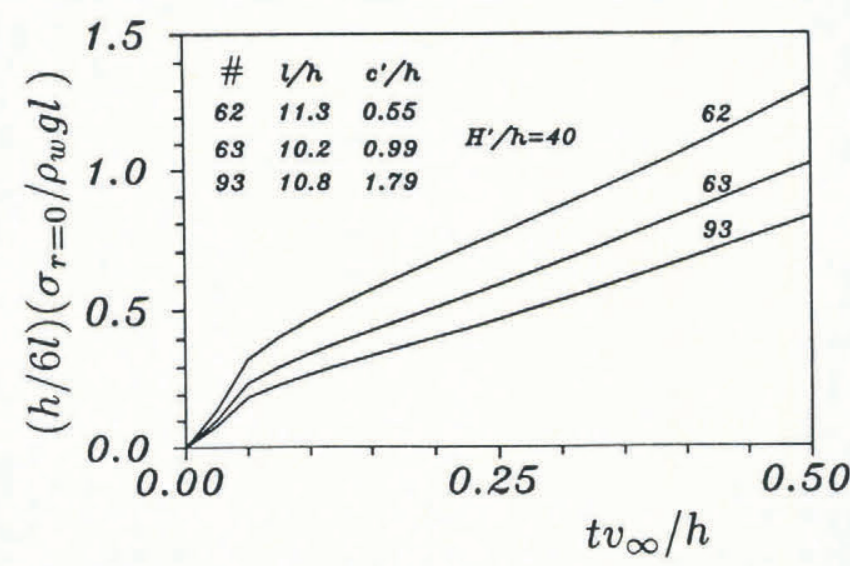

Fig. 5. Maximum radial bending stresses at $r^{\prime}=0$ versus time for test numbers 62, 63 and 93 in Sodhi's experiments. 
are approximately 582, 481 and $409 \mathrm{kPa}$, corresponding to test number $62\left(t v_{\infty} / h=0.43\right), 63\left(t v_{\infty} / h=0.46\right)$ and $93\left(t v_{\infty} / h=0.43\right)$. The associated flexural strengths found through cantilever tests in Sodhi's experiments were 60,60 and $67 \mathrm{kPa}$, respectively. Unfortunately, the maximum bending stresses seem to be over-predicted by an order of magnitude for the case of concentrated loadings. Note, however, that the experimentally determined maximum bending stresses are found via quasistatic uniaxial bending experiments. These could well be expected to differ significantly from strengths operative under conditions of sudden biaxial loadings.

An additional possibility, given the magnitudes of the differences observed, is that the plasticity of the ice sheet is affecting the results significantly. This was not expected, given the short times being considered. In this context, remember that the theoretically determined deflections $(\approx 0.45 h)$ corresponding to the uplift failure loads for three particular tests were much lower than the associated deflections recorded by Sodhi (1989).

An alternative possibility, supported by the physical evidence, involves a sequential bending failure mode. Since the theoretical solution indicates that the bending strengths are exceeded very early on at $r=0$, it is highly likely that the bending strengths are then exceeded at some radius greater than that of the uplifting cylinder. This supposition is further supported by the deflected shapes plotted by Sodhi (1989) starting with times corresponding closely to deflections at $r^{\prime}=c^{\prime}$ of half the ice-sheet thickness (the deflected shapes drawn by Sodhi exhibit what looks like the formation of a circumferential hinge).

Figure 6 shows the average shear stresses versus radial distance in different times for three test numbers. The average shear stresses at $r^{\prime}=c^{\prime}$ are approximately 61,38 and $24 \mathrm{kPa}$ corresponding to test number $62\left(t v_{\infty} / h=\right.$ 0.43), $63\left(t v_{\infty} / h=0.46\right)$ and $93\left(t v_{\infty} / h=0.43\right)$. Using a static formulation for the average shear stress at $r^{\prime}=c^{\prime}$

$$
\tau_{\max }=P_{\text {fail }}(2 \pi c h)^{-1},
$$

the corresponding values for the above three cases would be 89,47 and $31 \mathrm{kPa}$, respectively. The shear strengths recorded by Sodhi's quasi-static shear experiments are very close to the results from Equation (20). The aver-

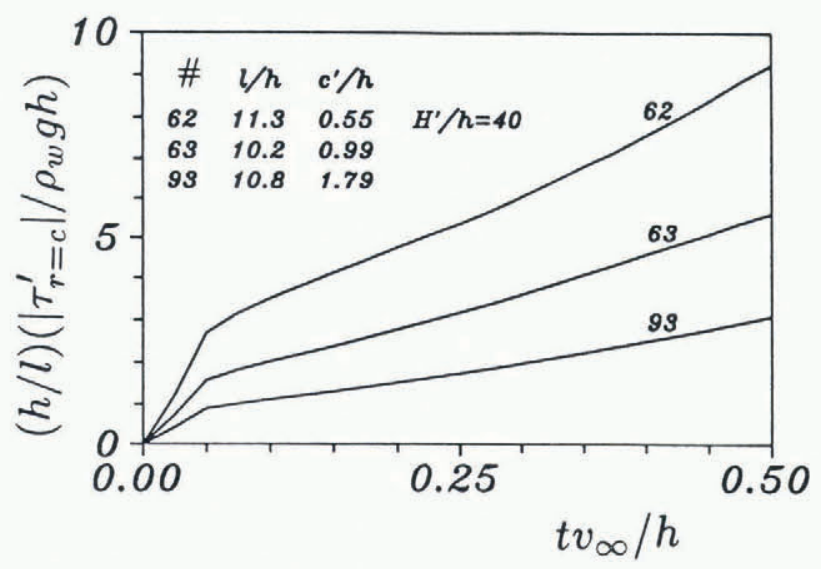

Fig. 6. Average shear stresses at $r^{\prime}=c^{\prime}$ versus time for test numbers 62, 63 and 93 in Sodhi's experiments. age shear stresses predicted by the present solution are smaller than the static solution since a certain part of the uplift load is balanced by hydrodynamic forces.

\section{CONCLUSIONS}

The transient response of an infinite ice sheet subjected to sudden axisymmetric uplift has been determined. For early times, the critical time duration, there is no validity to approximating the hydrodynamic plate foundation as a Winkler foundation. The bottom-surface pressure must be approximated by using incompressible potential flow theory. The theoretical solution in itself is useful as a guide for experimentalists as to the influence of the uplift rate, the characteristic length, the radius of the uplifting device and the thickness of the ice sheet. Comparisons with Sodhi's experiments revealed qualitatively similar behavior; quantitative comparisons suffered because:

- urea model ice was used in the test basin at CRREL by Sodhi (1989): this ice type is not idcally described by an undamped time-independent formulation;

- the characteristic length $l$ plays an influential role in the analysis, yet it is measured under quasi-static conditions only;

- the analysis treats an infinite ice sheet subjected to axisymmetric deformations, whereas the experiments were conducted in a finite and rectangular tank this disparity, however, is not expected to be significant;

- the loading by the flat-ended cylinder is approximated by a time-dependent ring load, which is highly idealized for very early times;

- not only was the bending strength exceeded at the center of the loaded region prior to uplift breakthrough, but also the former failure was followed by a circumferential bending failure at some radius greater than that of the cylinder radius;

- the average peak shear stresses were lower than the quasi-static strengths and, therefore, true shear failure probably never occurred since higher strengths would be expected for transient loading conditions; instead, the circumferential bending radius simply drifted closer to $r^{\prime}=c^{\prime}$ as the uplifting cylinder radius was made smaller.

\section{ACKNOWLEDGEMENTS}

The authors thank D.E. Nevel and D.S. Sodhi for several informative and interesting discussions. The research is supported in part by the U.S. Army through Grant DACA $98-88-\mathrm{K}-0013$ and in part by the National Science Foundation through Grant MSM 86-18798.

\section{REFERENCES}

Baker, T.H.C. 1977. The numerical treatment of integral equations. London, Oxford University Press.

Hertz, H. 1884. Über das Gleichgewicht schwimmender elastischer Platten. Wiedemann's Annalen der Physik und Chemie, 22,449-22,455.

Holl, D.L. 1950. Dynamic loads on thin plates on elastic foundations. In Proceedings of the 3rd AMS Symposium in Applied Mathematics. Vol. 3. New York, McGraw-Hill, 107-116. 
Dempsey and Zhao: Sub-surface uplift of floating ice sheet

Kheysin, D.Ye. 1967. Dinamica ledyanogo pokrova [Dynamics of the ice cover]. Leningrad, Gidrometeorologicheskoye Izdatel'stvo. (U.S. Technical Translation FSTC-HT-458-69.)

McGilvary, W.R., D.S. Sodhi, and J.H. Lever. 1990. Dynamic analysis of a floating ice sheet undergoing vertical indentation. In Ayorinde, O.A., N.K. Sinha, and D.S. Sodhi, eds. Proceedings of the Ninth International Conference on Offshore Mechanics and Arctic Engineering ... presented at ... Houston, Texas, February 18-23, 1990. Vol. 4. Arctic/polar technology. New York, American Society of Mechanical Engineers, 195-203.

Nevel, D. 1970. Vibration of a floating ice sheet. CRREL Res. Rep. 281.

Sodhi, D.S. 1989. Interaction forces during vertical penetration of floating ice sheets with cylindrical inden- tors. In Sinha, N.K., D.S. Sodhi, and J.S. Chung, eds. Proceedings of the Eighth International Conference on Offshore Mechanics and Arctic Engineering ... presented at ... The Hague, The Netherlands, March 19-23, 1988. Vol. 4. Arctic and polar technology. New York, American Society of Mechanical Enginecrs, 377-382.

Sodhi, D.S., K. Kato, F.D. Haynes, and K. Hirayama. 1982. Determining the characteristic length of model ice sheets. Cold Reg. Sci. Technol. 6(22), 99-104.

Westergaard, H.M. 1926. Stresses in concrete pavements computed by theoretical analysis. Public Roads, 7, 25-35.

The accuracy of references in the text and in this list is the responsibility of the authors, to whom queries should be addressed. 